\title{
A NOTE ON THE RELATIONSHIP BETWEEN SPECTRAL RADIUS AND NORMS OF BOUNDED LINEAR OPERATORS
}

\author{
H. M. Rodrigues* J. Solà-Morales ${ }^{\dagger}$
}

\begin{abstract}
Let $X$ be a Banach space and $\mathcal{L}(X)$ be the Banach algebra of bounded operators on $X$. In this note we prove that if we have a compact subset $K$ of a commutative sub-algebra of $\mathcal{L}(X)$, and given $\varepsilon>0$, then it is possible to define a new norm in $X$, equivalent to its given norm, in such a way that inside a neighborhood $U_{\varepsilon}$ of this compact set in the subalgebra, the norms of all the operators differ from their spectral radius in less than $\varepsilon$. If $X$ is a Hilbert space then it is possible to define this new norm as an Hilbertian norm.
\end{abstract}

When one wants to estimate the norms of functions of bounded operators the properties of norms on $\mathcal{L}(X)$ usually are very helpful. But imposing assumptions on the spectral radius of an operator is better than imposing on its norm, because the first is intrinsic and the second one is not. But for certain sets of bounded operators one can change the norm of the space to an equivalent norm in such a way that the norms of the bounded operators are as near as we wish to their spectral radius. This fact has been widely used by many authors, including C. Pugh classical paper [1, for example.

In our previous papers Rodrigues-Sola Morales [2] and [3] we also used this fact, and in 2] we proved that if we have a finite family of bounded linear operators $T_{n}$, such that $T_{n}$ commutes with $T_{m}$ for every $m, n$, then it is possible to define a new norm on the Banach space $X$, equivalent to the initial norm,

\footnotetext{
Mathematics Subject Classification. Primary 47A10, 47A30; Secondary 46J05

Key words and phrases. spectral theory, adapted norms, spectral radius.

* Partially supported by CNPq Proc.: 301994/85-4, Programa Pronex-Projeto Tematico, CNPq-FAPESP n. 2003/10042-0, FAPESP Proc.: 2005/55694-0, Projeto Milenio/IMPA Proc.: 420108/2005-0 and CAPES Proc.: 1356062, Brasil.

${ }^{\dagger}$ Partially supported by MEC, Spain (MTM2005-07660-C02-01) and by DURSI, Generalitat de Catalunya (2005BE00245).
} 
in such a way that in the new norm the spectral radius, $r\left(T_{n}\right)$, approximates the norm of the operator $T_{n}$, for every $n$. For just one operator this result was proved by Holmes [4. When the family consists of two operators, and of the form $T, T^{-1}$, the result had already appeared in the mathematical literature, like for example in Cabre, Fontich, de la Llave [6].

In the present note we improve the above result by proving that if we have a compact subset, $K$, of a commutative sub-algebra of $\mathcal{L}(X)$, and given $\varepsilon>0$, then it is possible to define a new norm on $X$, equivalent to its given norm, in such a way that in a neighborhood $U_{\varepsilon}$ of this compact set inside the subalgebra, the norms of all the operators differ from their spectral radius in less than $\varepsilon$. If $X$ is a Hilbert space then it is possible to define a new Hilbertian norm with the specified property. This answers a question posed by Charles Pugh to one of the authors.

In a Banach space $X$ with a norm $|\cdot|$, given an operator $T \in \mathcal{L}(X)$ and a number $\varepsilon>0$ one can define a new norm $|\cdot|_{T, \varepsilon}$ with the formula:

$$
|x|_{T, \varepsilon}=\left[|x|^{2}+\left(\frac{|T x|}{M}\right)^{2}+\cdots+\left(\frac{\left|T^{m} x\right|}{M^{m}}\right)^{2}\right]^{1 / 2},
$$

where $M=r(T)+\varepsilon$ and $m \in \mathbb{N}$ has been chosen as the first integer such that $\left|T^{m}\right|^{1 / m}<M$, where we denote by $r(T)$ the spectral radius of $T$. It is easy to see that this norm is equivalent to the original norm and that

$$
|T|_{T, \varepsilon}<r(T)+\varepsilon
$$

We are interested in pointing out the following property, that plays a key role in the rest of this paper:

Lemma 1. If $T, S \in \mathcal{L}(X)$ and $S T=T S$, then

$$
|S|_{T, \varepsilon} \leq|S|
$$

Proof: It is easy to see that $|S x|_{T, \varepsilon} \leq|S||x|_{T, \varepsilon}$, for every $x \in X$.

As a consequence of this simple lemma, one can prove the following: 
Proposition 1. If $T, S \in \mathcal{L}(X)$ and $S T=T S$, then

(a) $r(T+S) \leq r(T)+r(S)$ and $r(T S) \leq r(T) r(S)$.

(b) The spectral radius of $T \in \mathcal{L}(X)$ changes continuously when $T$ is subjected to a small change which commutes with $T$.

Proof: (a) Given $\varepsilon>0$, from Lemma 1, there exists a new norm $\|\cdot\|$, such that $\|T\|<r(T)+\varepsilon,\|S\|<r(S)+\varepsilon$, So

$$
\begin{gathered}
r(T+S) \leq\|T+S\| \leq\|T\|+\|S\|<r(T)+r(S)+2 \varepsilon \\
r(T S) \leq\|T S\| \leq\|T\|\|S\|<r(T) r(S)+\varepsilon(r(T)+r(S))+\varepsilon^{2} .
\end{gathered}
$$

(b) From (a) it follows that:

$$
r(T)=r(T-S+S) \leq r(T-S)+r(S) \leq\|T-S\|+r(S)
$$

and

$$
r(S)=r(S-T+T) \leq r(S-T)+r(T) \leq\|T-S\|+r(T)
$$

Therefore $-\|T-S\| \leq r(T)-r(S) \leq\|T-S\|$. This implies the continuity.

The following is our main result:

Theorem 1. Let $X$ be a Banach space with a norm $|\cdot|$ and let $K$ be a compact subset of $\mathcal{L}(X)$ such that $T S=S T$ for all $T, S \in K$.

(i) Given $\varepsilon>0$ it is possible to define a new norm $\|\cdot\|$ equivalent to the given norm $|\cdot|$ in such a way that

$$
\|T\|<r(T)+\varepsilon,
$$

for every $T \in K$.

(ii) Given a set $\mathcal{U}$, not necessarily compact, with $K \subset \mathcal{U} \subset \mathcal{L}(X)$ and such that $T S=S T$ for all $T, S \in \mathcal{U}$, property 0.1 also holds for $T$ in a neighborhood $U_{\varepsilon}$ of $K$ inside $\mathcal{U}$. 
Proof: For all $T \in K$ we consider the open ball centered at $T$ of radius $\varepsilon / 3$. This is a covering of $K$, from which one can extract a finite covering, with centers $T_{1}, T_{2}, \cdots, T_{n}$.

By using the above procedure, we modify successively the norm $|\cdot|$ of $X$ to $|\cdot|_{T_{1}, \varepsilon / 3}$, then by using the operator $T_{2}$ we modify the norm $|\cdot|_{T_{1}, \varepsilon / 3}$ and so on.

At the end, we have an equivalent norm $\|\cdot\|$, such that, by Lemma 1 .

$$
\left\|T_{i}\right\|<r\left(T_{i}\right)+\varepsilon / 3
$$

for $i=1,2, \cdots, n$ and such that $\|S\| \leq|S|$ for all $S \in \mathcal{U}$.

Given now any $T \in K$, there exists a $T_{j}$ such that $\left|T-T_{j}\right|<\varepsilon / 3$, and we have:

$$
\|T\| \leq\left\|T_{j}\right\|+\left\|T-T_{j}\right\| \leq\left\|T_{j}\right\|+\left|T-T_{j}\right|
$$

where we have used that $T-T_{j}$ commutes with all of the $T_{i}$ 's. And now

$$
\|T\| \leq\left\|T_{j}\right\|+\varepsilon / 3<r\left(T_{j}\right)+\varepsilon / 3+\varepsilon / 3 .
$$

Finally, by Proposition 1 .

$$
r\left(T_{j}\right) \leq r(T)+r\left(T-T_{j}\right) \leq r(T)+\left|T-T_{j}\right|<r(T)+\varepsilon / 3
$$

Then $\|T\|<r(T)+\varepsilon$ and the proof or (i) is complete.

To prove part (ii) we first use part (i) and consider the norm $\|\cdot\|$ such that

$$
\|T\|<r(T)+\varepsilon
$$

for all $T \in K$. Then we observe that because of Proposition 1 the map $T \mapsto$ $r(T)$ is continuous from $\mathcal{U}$ to $\mathbb{R}$, where $\mathcal{U}$ inherits the topology of the norm $\|\cdot\|$ of $X$. Then it is obvious that the set

$$
\{T \in \mathcal{U}:\|T\|-r(T)<\varepsilon\}
$$

contains $K$ and is an open set in the relative topology of $\mathcal{U}$. 
Remark 1. The previous results hold true without changing to a new norm if $X$ is a Hilbert space and $T$ is a selfadjoint or a normal operator.

Remark 2. The result (ii) needs not to be true if one drops the property that $T S=S T$ for all $T, S \in \mathcal{U}$, that is if we speak about the neighborhoods of $K$ in $X$. As it is explained in Kato [5] the spectral radius can shrink discontinuously in arbitrary neighborhoods of an operator.

Remark 3. Note that every compact $K \subset \mathcal{L}(X)$ as in Theorem 1 generates a commutative Banach algebra $\mathcal{A} \subset \mathcal{L}(X)$, so the set $\mathcal{U}$ could be taken as $\mathcal{U}=\mathcal{A}$.

Let us explain some more what could be one of the uses of Theorem 1 above. If $X$ is a Banach space, and $L \in \mathcal{L}(X)$, then one can consider the operator $f(L)$ defined with the Operational Calculus formula:

$$
f(L)=\int_{\gamma} f(z)(L-z I)^{-1} d z .
$$

The typical case is when $f$ is an holomorphic function in an open set $\Omega \subset \mathbb{C}$ with $\sigma(L) \subset \Omega$ and $\gamma:[0,1] \rightarrow \Omega \backslash \sigma(L)$ is a closed rectificable curve. Then, the set

$$
K=\left\{(L-z)^{-1} \mid z \in\{\gamma\}\right\} \subset \mathcal{L}(X)
$$

is a compact set of commuting operators.

Then the previous Theorem can be applied, and given an arbitrary $\varepsilon>0$ there exists a suitable new equivalent norm || || such that

$$
\|f(L)\| \leq \int_{0}^{1}\left|\gamma^{\prime}(s)\right| \frac{|f(\gamma(s))|}{\operatorname{dist}(\gamma(s), \sigma(L))} d s+\varepsilon \text { lenght }[\gamma],
$$

an inequality that can be useful in many cases. And we point out that the inequality 0.3 , and inequalities of similar type, can be derived from 0.2 without requiring $f(z)$ to be holomorphic in $\Omega$ containing $\sigma L$. The only thing that one needs is $f(\gamma(s))$ to be integrable in $(0,1)$.

Some of the above results can be extended to abstract Banach algebras. 
Theorem 2. Let $\mathcal{A}$ be a commutative algebra of Banach with norm $|\cdot|$. Let $K$ be a compact subset of $\mathcal{A}$. Then given $\varepsilon>0$, there exists a new norm $\|\cdot\|$ in $\mathcal{A}$ equivalent to $|\cdot|$ and a neighborhood $U_{\varepsilon}$ of $K$, such that for every $T \in U_{\varepsilon}$

$$
\|T\|<r(T)+\varepsilon .
$$

Proof: The proof follows the ideas of Theorem 1 with minor changes. We first observe that $\mathcal{A}$ with norm $|\cdot|$ is a Banach space. For $T \in \mathcal{A}$, we define a new Banach space norm as:

$$
|S|_{T, \varepsilon}=\left[|S|^{2}+\left(\frac{|S T|}{M}\right)^{2}+\cdots+\left(\frac{\left|S^{m} T\right|}{M^{m}}\right)^{2}\right]^{1 / 2},
$$

where $M$ and $m$ are given as above. This norm will induce a Banach algebra norm, $|\cdot|_{T, \varepsilon}$, in $\mathcal{L}(\mathcal{A})$, defined in the usual way and $\mathcal{A} \subset \mathcal{L}(\mathcal{A})$.

It is possible to prove that this norm restricted to $\mathcal{A}$ is equivalent to the norm $|\cdot|$.

The remaining part of the proof follows the steps of the proof of Theorem 1.

\section{References}

[1] Pugh, C. C., On a Theorem of P. Hartman, American Journal of Mathematics 91, 2, (1969), 363-367.

[2] Rodrigues, H. M.; Solà-Morales, J., Linearization of Class $C^{1}$ for Contractions on Banach Spaces, J. Differential Equations 201, 2, (2004), 351-382.

[3] Rodrigues, H. M.; Solà-Morales, J., Smooth Linearization for a saddle on Banach Spaces, J. Dynamics and Differential Equations 16, 3,(2004), 767793.

[4] Holmes, R. B., A formula for the spectral radius of an operator, Amer. Math. Monthly 75 (1968), 163-166. 
[5] Kato, T., (1976). Perturbation Theory for Linear Operators, Second Edition, Springer Verlag, 1976.

[6] Cabré, X.; Fontich, E.; de la Llave, R., The parametrization method for invariant manifolds, I. Manifolds associated to non-resonant subspaces. Indiana Univ. Math. J. 52, 2, (2003), 283-328.

H. M. Rodrigues

Departamento de Matemática Aplicada e Estatística

Instituto de Ciências Matemáticas e de Computação

Universidade de São Paulo

Caixa Postal 668, 13560-970

São Carlos, SP, Brazil

E-mail: hmr@icmc.usp.br

\section{J. Solà-Morales}

Departament de Matemàtica

Aplicada 1

Universitat Politècnica de Catalunya

Av. Diagonal 647

08028 Barcelona, Spain

E-mail: jc.sola-morales@upc.edu 